\title{
Hybrid Point Cloud Semantic Compression for Automotive Sensors: A Performance Evaluation
}

\author{
Andrea Varischio, Francesco Mandruzzato, Marcello Bullo \\ Marco Giordani, Paolo Testolina, Michele Zorzi \\ Department of Information Engineering, University of Padova, Padova, Italy \\ \{name.surname\}@dei.unipd.it
}

\begin{abstract}
In a fully autonomous driving framework, where vehicles operate without human intervention, information sharing plays a fundamental role. In this context, new network solutions have to be designed to handle the large volumes of data generated by the rich sensor suite of the cars in a reliable and efficient way. Among all the possible sensors, Light Detection and Ranging (LiDAR) can produce an accurate 3D point cloud representation of the surrounding environment, which in turn generates high data rates. For this reason, efficient point cloud compression is paramount to alleviate the burden of data transmission over bandwidth-constrained channels and to facilitate real-time communications. In this paper, we propose a pipeline to efficiently compress LiDAR observations in an automotive scenario. First, we leverage the capabilities of RangeNet++, a Deep Neural Network (DNN) used to semantically infer point labels, to reduce the channel load by selecting the most valuable environmental data to be disseminated. Second, we compress the selected points using Draco, a 3D compression algorithm which is able to obtain compression up to the quantization error. Our experiments, validated on the Semantic KITTI dataset, demonstrate that it is possible to compress and send the information at the frame rate of the LiDAR, thus achieving real-time performance.
\end{abstract}

Index Terms-Autonomous driving, compression, Draco, Deep Neural Networks (DNNs), RangeNet++, point cloud, LiDAR.

\section{INTRODUCTION}

Light Detection and Ranging (LiDAR) sensors play a crucial role in the field of autonomous driving, providing vehicles with a thee-dimensional (3D) omnidirectional perception of the surrounding environment [1]. Compared to other types of sensors, such as automotive cameras and radars, LiDAR data is represented in the form of large point clouds carrying both geometry and attribute information (e.g., depth properties), to achieve fine detection, localization, and recognition of the surrounding objects [2]. However, LiDAR point clouds may generate very large volumes of data. For example, a 3D frame with 0.7 million points acquired at $30 \mathrm{fps}$ needs a bandwidth of around $500 \mathrm{Mbps}$ [3], which can be challenging to handle with standard communication systems [4] (e.g., the IEEE $802.11 \mathrm{p}$ transmission service, currently employed for Vehicle-to-Vehicle (V2V) operations, offers data exchange at a nominal rate up to 27 Mbps [5]).

One possible approach to solve capacity issues is by developing new radio systems, e.g., operating in the lower part of the millimeter wave (mmWave) spectrum, as currently promoted by IEEE and 3GPP standardization activities for next- generation vehicular networks [6]. This potential is however hindered by the harsh propagation characteristics of the above$6 \mathrm{GHz}$ bands, which might be particularly critical in the dynamic vehicular environment [7].

Data rates can be further reduced by compressing the point clouds in a sufficient ratio and make them suitable for realtime transmission. In this context, 2D-oriented compression techniques, such as JPG or PNG, might be ineffective in the 3D space as point clouds, unlike images, are sparse and nongrid structured. The most common way to structure 3D data is indeed to enclose points in a bounding cube and discretize the content in a so-called voxel-grid, thus obtaining a threedimensional matrix at a specific resolution.

Following this intuition, several literature works have approached 3D compression by using data structures such as Octrees [8], which could not however detect and appropriately remove redundant information hidden in the point cloud representations. More recently, novel techniques based on deep learning have made it possible to further improve point cloud compressibility by extracting features from individual points and predicting voxel occurrence in 3D scenes, e.g., [9], [10].

Based on the above introduction, in this paper we aim at improving the compression efficiency of LiDAR point clouds by combining the compression capabilities of the Google software Draco [11] with the semantic segmentation functionalities provided by RangeNet++ [12], a Deep Neural Network (DNN) used to assign class labels to data points. As illustrated in Fig. 1, the proposed Hybrid Semantic Compression (HSC) pipeline works as follows:

1) We first infer full semantic segmentation of LiDAR point clouds with RangeNet++, so as to identify the most valuable objects in the scene (typically pedestrians and vehicles). If capacity is limited, this allows deferring or canceling the transmissions of the least critical acquisitions [13], [14], while prioritizing the most important LiDAR frames.

2) We compress the resulting point cloud with Draco, which defines 15 quantization levels to consider different encoding/decoding speeds and discretization losses.

The performance of the proposed HSC framework is evaluated in terms of average compression ratio (an indication of how efficiently data is compressed), Peak Signal to Noise Ratio (PSNR), and symmetric Chamfer Distance (used to measure the quality of the reconstructed point cloud). For 


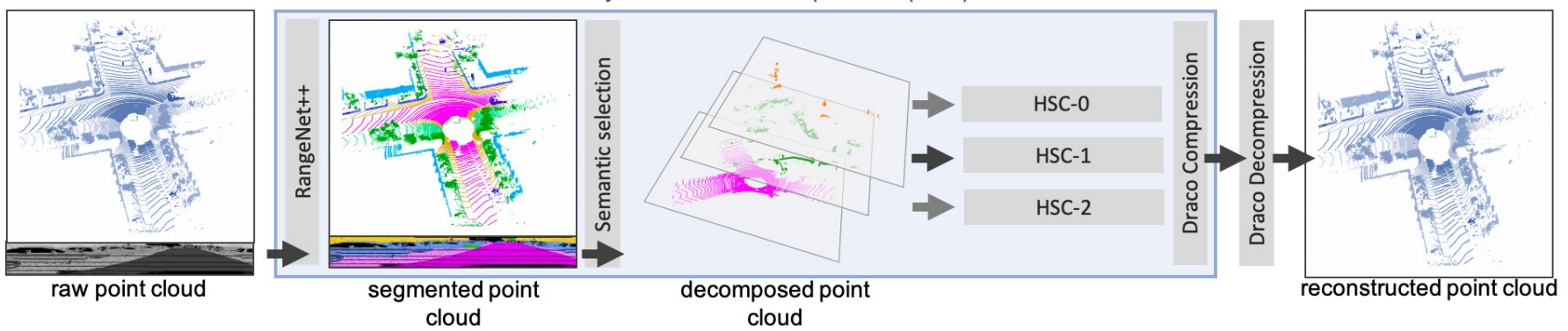

Fig. 1: An overview of the proposed Hybrid Semantic Compression (HSC) pipeline for LiDAR point clouds.

completeness, our approach is compared with a baseline geometry-based point cloud compression (G-PCC) scheme which is able to handle sparse data working directly on the 3D space [15]. Our results, which are validated on the Semantic KITTI dataset [16], show that HSC can deliver quasi-real-time compression performance with limited accuracy degradation, especially when designing aggressive semantic segmentation.

The rest of this paper is organized as follows. Sec. II concisely describes related works on point cloud compression. Sec. III and Sec. IV provide a brief overview of RangeNet++ and Draco, respectively. In Sec.V]we present our experimental results, whereas Sec. VI concludes the article.

\section{RELATED WORK}

The adoption of LiDARs on commercial vehicles triggered new interest in 3D point cloud compression. Prior works, e.g., [17], [18], first addressed this research challenge by projecting the $3 \mathrm{D}$ point cloud into a $2 \mathrm{D}$ representation to perform bidimensional compression, achieving real-time performance. Despite their desirable computational speed, however, such methods were proven to be inefficient when applied to dense 360-degree LiDAR point clouds.

In recent times, the introduction of deep learning in networks allowed researchers to broaden their horizons, and design more sophisticated compression schemes based on the automatic extraction of features from data. In [19], for example, Zhou et al. introduced an end-to-end trainable deep network to extract features automatically from equally spaced 3D voxel structures, and to make bounding box prediction based on them. However, the sparsity of the 3D LiDAR data resulted in many empty voxel cells, thus making this representation inefficient. In [10] the authors designed a neural network to process point clouds while being invariant to input permutations. Despite its inherent simplicity, this method performs poorly on large LiDAR point clouds, and the memory required to store such representations is prohibitively large. Some other studies have introduced auto-encoders or recurrent neural networks that directly work on the 3D geometry. Specifically, in [20] variational auto-encoders, in combination with a scale hyperprior, have been exploited to capture spatial dependencies in the latent space. The drawback of such models is that they require very complex learning capabilities, especially with sparse data structures, which may be difficult to guarantee on board of vehicles.

Research efforts have then been dedicated to the design of compression strategies operating on data structures able to accurately represent point clouds. One of the most popular methods is based on tree structures, like Octree or KD-tree. The advantage is their ability to model arbitrary point clouds in a hierarchical way, dealing also with sparsity. For example, in [9] point clouds are first stored in an Octree and then the extracted context features are fed into a deep entropy model to be encoded into a compact bitstream.

Compression can be further improved when a full characterization of the environment is provided, as described in [12], [21], [22], which is the main idea behind the proposed HSC framework.

\section{RANGENET++}

In this section we provide a brief description of RangeNet++ [12], which is used to assign a class label to 3D LiDAR points, an essential step in HSC to filter the raw point cloud keeping only the most relevant/critical points. For a more complete description of the RangeNet++ network we refer the interested reader to [12].

RangeNet++ is a deep convolutional neural network able to accurately perform semantic segmentation using only the LiDAR point cloud and its reflectance. Furthermore, it can infer the point labels without discarding any point, while working at the frame rate of the LiDAR scanner.

The raw 3D data is first mapped on a range image through a spherical projection. Features are then extracted from the range representation and fed into an encoder-decoder architecture. After inferring the labels (e.g., road, pedestrian, vegetation, etc.) for the current frame, each point is reprojected to the original point cloud. A k-nearest neighbor $(\mathrm{kNN})$ search is then employed to reduce the blurring introduced by the network and increase the segmentation accuracy.

As reported in [12], the size of the range image affects the accuracy of the segmentation and the number of frames processed per second. In this work, we set the size of the image resolution to $[64 \times 2048]$, which is the maximum resolution supported by RangeNet++, and was reported to provide very accurate results even with limited power resources [12]. 


\section{DRACO}

LiDAR acquisitions are very sparse point clouds without a pre-defined structure. In this context, Draco [11], a software designed by Google, uses a KD-tree data structure [23] to efficiently organize 3D data while dealing with sparsity, and provides compression at very high speed.

Despite its wide adoption in the literature, the software lacks a detailed documentation. In this work, we just give an idea of how it works. Unlike Octree-based solutions, Draco keeps on splitting the point cloud from the center, alternating the axes at each iteration in order to build a more balanced space-partitioning tree. The number of points after each split operation is encoded as the difference between the number of points contained in the smaller half of the point cloud and the total number of points, divided by two. This results in more leading zeros in the encoded values, which are better compressed by arithmetic encodings.

Draco's flexibility allows to define 15 quantization levels $(0-14)$ and 11 compression levels $(0-10)$ that trade off the compression efficiency against its speed. Compression accuracy is then proportional to the quantization error: as the quantization level grows, the compression quality increases at the cost of a larger file, whereas higher quantization levels translate in more compression but lower decompression speed.

\section{COMPRESSion Performance Results}

In this section we first overview our performance metrics (Sec. V-A), then describe the G-PCC architecture, which is used to benchmark the performance of the proposed HSC framework (Sec. $\mathrm{V}-\mathrm{B}$ ), and finally present our main performance results (Sec. $\mathrm{V}-\mathrm{C}$ ).

To evaluate the compression performance of HSC we use the Semantic KITTI dataset [16], where LiDAR sensors generate point clouds of $120^{\prime} 000$ points per frame, on average every $100 \mathrm{~ms}$. HSC has been designed to support three possible transmission levels:

- HSC-0: The raw LiDAR acquisition is immediately compressed by Draco and sent through the channel. In this case, RangeNet++ is not used.

- HSC-1: RangeNet++ is used to perform semantic segmentation on the raw LiDAR acquisitions, as depicted in Fig. 2. After that, the points belonging to the road elements are removed from the point cloud, thus reducing the file size. This choice can be justified by assuming the availability of topology information from auxiliary data sources such as Google Maps or similar mapping tools.

- HSC-2: Compared to HSC-1, buildings, vegetation, and traffic signs, which may be provided by camera sensors, if available, are also removed from the point cloud after RangeNet++ segmentation. Consequently, the resulting point cloud to be compressed consists only of dynamic elements like pedestrians and vehicles, i.e., the most critical elements in autonomous driving scenarios [24].

It is important to highlight the versatility of the proposed pipeline: the segmentation performed by RangeNet++

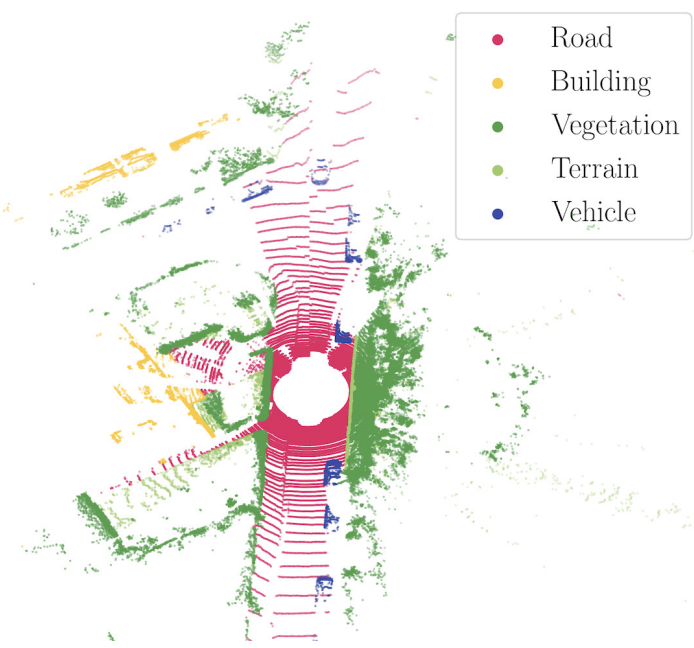

Fig. 2: Example of point cloud semantic segmentation performed by RangeNet++. Each set of points may or may not be removed from the raw LiDAR point cloud before compression, depending on how aggressively the HSC framework is configured.

can be exploited during the transmission phase to prioritize safety-critical over less relevant information. Consider, for instance, the User Datagram Protocol (UDP), a common choice at the transport layer for time-sensitive applications. UDP's lean design permits fast data packet transmissions, while in turn suffering from lossy connection, which may require full retransmission of packets. Moreover, UDP has a maximum packet size of $65^{\prime} 535$ Bytes, which poses a significant constraint on the dimension of the LiDAR data to avoid fragmentation, even after compression. In light of this, data selection plays a vital role in this context. In the HSC framework, the opportunity to remove from raw LiDAR point clouds nonessential static elements after RangeNet++ segmentation (i.e., in HSC-1 and HSC-2) makes it possible to encode higher-priority information in the same UDP packets, which are to be transmitted first.

\section{A. Performance Metrics}

In this section we present the metrics used to evaluate the performance of the investigated compression solutions.

a) Bits per point $(B P P)$ : This quantity represents the number of bits used to compress each point in the original point cloud, and is computed as the ratio between the compressed file size and the number of points in the compressed file. A larger BPP would result in a better quality of the reconstructed point cloud, at the cost of a lager memory footprint.

b) Average compression ratio: This metric indicates the relative reduction in size of the data representation produced by compression, and is measured as the ratio between the raw point cloud size and the compressed file size.

c) Symmetric point-to-point Chamfer Distance: To evaluate the quality of the reconstructed point cloud $\hat{P}$ with respect to the raw point cloud $P$, we adopt the symmetric point-topoint Chamfer Distance $\mathrm{CD}_{\text {sym }}$, which is defined as

$$
\mathrm{CD}_{\text {sym }}=\sum_{\forall \mathbf{p} \in P} \min _{\forall \hat{\mathbf{p}} \in \hat{P}}\|\mathbf{p}-\hat{\mathbf{p}}\|_{2}^{2}+\sum_{\forall \hat{\mathbf{p}} \in \hat{P}} \min _{\forall \mathbf{p} \in P}\|\mathbf{p}-\hat{\mathbf{p}}\|_{2}^{2} .
$$


As $\mathrm{CD}_{\text {sym }}$ approaches zero, the reconstructed point cloud, $\hat{P}$, better approximates the original one, $P$.

d) Point-to-plane PSNR: Given a point $\boldsymbol{p} \in P$ and its nearest neighbor $\boldsymbol{q} \in \hat{P}$, the point-to-plane Mean Square Error (MSE) computed with respect to $P$ is

$$
\mathrm{MSE}_{P \rightarrow \hat{P}}=\frac{1}{|P|} \sum_{\forall \boldsymbol{p} \in P}\left(\left\langle\boldsymbol{p}-\boldsymbol{q}, \boldsymbol{n}_{\boldsymbol{q}}\right\rangle\right)^{2},
$$

where $\boldsymbol{n}_{\boldsymbol{q}}$ is the surface normal in $\boldsymbol{q} \in \hat{P},|P|$ is the cardinality of the point cloud $P$ and $\left\langle\boldsymbol{p}-\boldsymbol{q}, \boldsymbol{n}_{\boldsymbol{q}}\right\rangle$ is the projection of vector $\boldsymbol{p}-\boldsymbol{q}$ on $\boldsymbol{n}_{\boldsymbol{q}}$. Then, the point-to-plane Peak Signal to Noise Ratio (PSNR) with respect to $P$ is given by

$$
\operatorname{PSNR}_{P \rightarrow \hat{P}}=10 \log _{10}\left(\frac{\left(\gamma_{P}^{*}\right)^{2}}{\operatorname{MSE}_{P \rightarrow \hat{P}}}\right)
$$

where $\gamma_{P}^{*}$ is a peak value in the point cloud $P$. Conventionally, the peak value $\gamma_{P}^{*}$ is defined based on the diagonal distance of a bounding box of the point cloud $P$, which would however imply that higher PSNR is produced when larger objects are considered, thus resulting in an unbalanced metric. To solve this issue, we compute $\gamma_{P}^{*}$ using the intrinsic resolution of the point cloud $P$, as proposed in [25]. In practice, $\gamma_{P}^{*}$ is derived from the nearest neighbor distances $d_{p}$ for all points $\mathbf{p}$ in the input point cloud $P$, i.e., $\gamma_{P}^{*}=\max _{\forall p \in P}\left\{d_{p}\right\}$. Finally, the symmetric PSNR between $P$ and $\hat{P}$ is defined as

$$
\operatorname{PSNR}_{P, \hat{P}}=\min \left\{\operatorname{PSNR}_{P \rightarrow \hat{P}}, \operatorname{PSNR}_{\hat{P} \rightarrow P}\right\} .
$$

\section{B. Compression Baseline: $G-P C C$}

The MPEG group is developing a new standard for point cloud compression called G-PCC (also known as TMC13) [15]. The G-PCC codec algorithm, like Draco, works directly in the 3D space and allows to code both geometry and attributes of a point cloud following two parallel approaches. In particular, G-PCC voxelizes the raw point cloud by quantizing coordinates to closest integer. Furthermore, translation and scaling transformations are applied such that the resulting point cloud resides in a bounding cube of size $\left[0,2^{d}\right]^{3}$, for some non negative integer $d$ defined as:

$$
d=\left\lceil\left(\log _{2}\left[\max \left(x_{n}^{\text {int }}, y_{n}^{\text {int }}, z_{n}^{\text {int }}\right)+1\right)\right]\right\rceil, \forall n=1, \ldots, N,
$$

where $\left[x_{n}^{\text {int }}, y_{n}^{\text {int }}, z_{n}^{\text {int }}\right]$ are the point cloud's 3D coordinates, $N$ is the total number of points in the cloud, and $\lceil\cdot\rceil$ is the ceiling function. The processed point clouds are then stored in an Octree-based data structure and finally arithmetic coding is applied. Notice that the quantization performed by G-PCC may collapse many points into the same position depending on the spatial resolution allowed in the voxel grid. On this work, the scaling factor has been tuned so duplicate points are avoided.

\section{Experimental Results}

In the following results we compare the compression efficiency (in terms of file size, compression ratio and processing time) and accuracy (in terms of Chamfer Distance and PSNR) of HSC vs. a baseline G-PCC implementation. All our

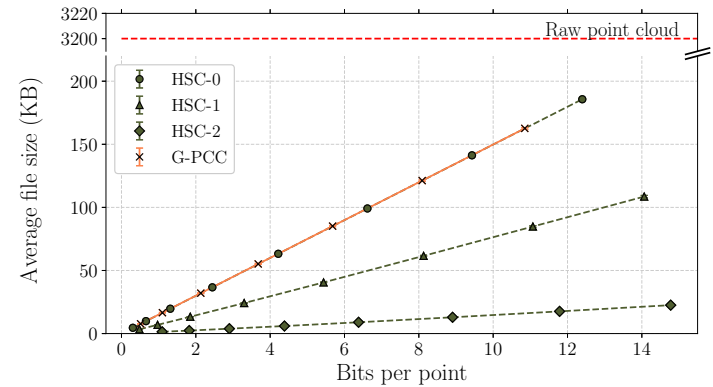

Fig. 3: File size vs. the BPP, as a function of the compression strategy.

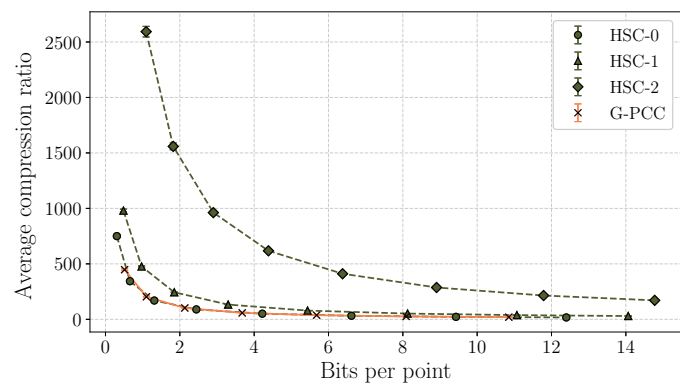

Fig. 4: Average compression ratio vs. the BPP, as a function of the compression strategy.

tests were performed on an NVIDIA ${ }^{\mathrm{TM}}$ TITAN $^{\circledR}$ RTX GPU, equipped with an Intel ${ }^{\circledR}$ Core $^{\mathrm{TM}}$ i7-7700K CPU at $4.20 \mathrm{GHz}$ and $32 \mathrm{~GB}$ of RAM. The metrics are shown as a function of the BPP used to compress each point in the raw point cloud, as defined in Sec. V-A. Specifically, the value of the BPP depends on how aggressive the compression is, i.e., on the adopted quantization level (for HSC) and the voxelization scaling factor (for G-PCC).

Compression efficiency. In Fig. 3 we show the average file size obtained with HSC and G-PCC. In general, it is necessary to reduce the file size as much as possible to keep the channel free for transmissions generated from sensory sources other than the LiDAR, or to serve high-priority traffic requests (e.g., in case of emergency). It is evident that, in fact, even considering only LiDAR transmissions, standard vehicular communication technologies would not be able to satisfy traffic requests efficiently, thus creating congestion: from Fig. 3, raw LiDAR perceptions of $3200 \mathrm{~KB}$, generated every $100 \mathrm{~ms}$, would require a data rate of at least 256 Mbps, which is far beyond what current IEEE 802.11p implementations for $\mathrm{V} 2 \mathrm{~V}$ communications can support. Notably, the HSC framework can compress the raw LiDAR point cloud up to 8 times more effectively than G-PCC. Moreover, unlike Draco (i.e., HSC-0) and G-PCC, HSC-2 allows to encapsulate the semantically inferred point cloud in a single UDP packet of 65 '535 Bytes regardless of the quantization level, a fundamental pre-requisite to guarantee that the most critical and time-sensitive data reaches the receiver(s) with the lowest latency and the highest reliability. Similarly, this is possible using HSC-1 with less than $10 \mathrm{BPP}$. 


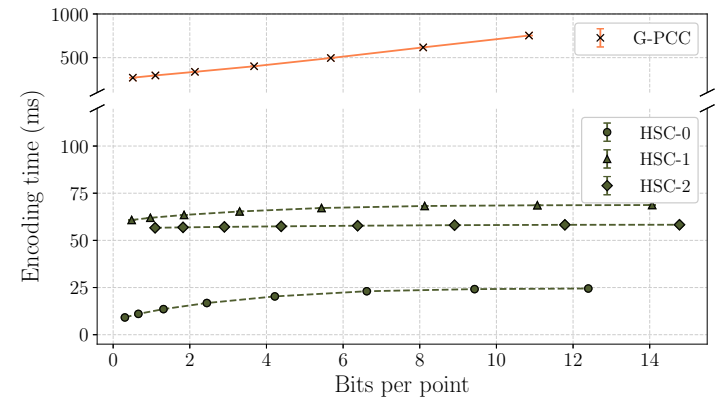

(a) Encoding time.

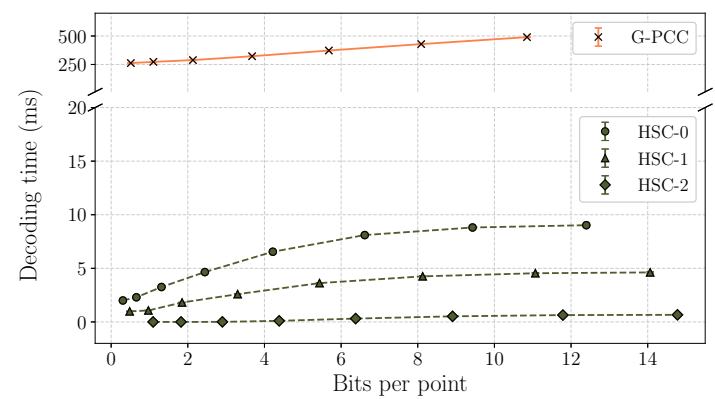

(b) Decoding time

Fig. 5: Encoding and decoding time of the investigated compression strategies vs. the BPP. For HSC-1 and HSC-2, these metrics also account for the average time required by RangeNet++ to semantically infer labels to point clouds.

The same conclusions can be derived from Fig. 4, which illustrates the average compression ratio vs. the BPP. As expected, not only can HCP provide better compression compared to G-PCC, but semantic segmentation before compression (i.e., HSC-1 and HSC-2) can also improve the compression efficiency by more than 10 times with respect to a standard Draco-only approach (i.e., HSC-0) by removing non-critical data before transmission.

In terms of computational efficiency, Fig. 5 shows that, as the BPP increases, the quantization is proportionally less aggressive and, consequently, both encoding and decoding times increase significantly. Regarding G-PCC, the time required to encode and decode a point cloud is orders of magnitude higher than in HSC, which validates the inefficiency of representing $3 \mathrm{D}$ data in an Octree-based structure compared to a KDtree, respectively. Considering a LiDAR frame rate of 100 ms, Fig. 5 reports that only the HSC framework can encode and decode within the LiDAR inter-frame time even with the highest quantization level.

Fig. 5a also exemplifies that, in HSC-1 and HSC-2, the RangeNet++ inference time must be considered in addition to that of Draco, namely $56 \mathrm{~ms}$ on average using the hardware specified in Sec. V-C. This preprocessing time could be cut down if considering half of the range image resolution for RangeNet++ (i.e., $[64 \times 1024]$ ), at the cost of reducing the Intersection over Union (IoU), which is used to measure the object detection accuracy in a particular scene. The encoding time can be further decreased if the burden of segmentation is

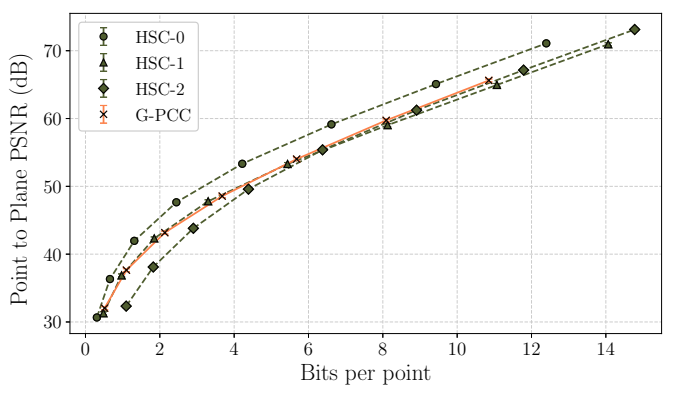

Fig. 6: PSNR of the investigated compression strategies vs. the BPP.

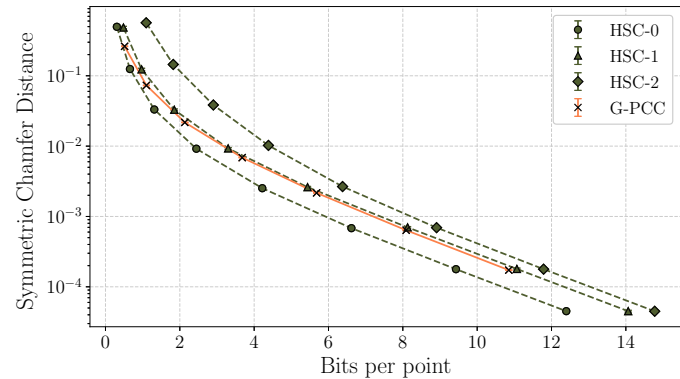

Fig. 7: Symmetric point-to-point Chamfer Distance of the investigated compression strategies vs. the BPP.

delegated to more powerful cloud computing facilities before transmission. On the contrary, decoding operations should be executed on-board to ensure real-time responses, thereby making the proposed HSC-1 and HSC-2 schemes (which are faster than HSC-0 and G-PCC) more desirable, as shown in Fig. $5 b$

Compression accuracy. Looking at the PSNR in Fig. 6 HSC-0 is able to reach the same accuracy of G-PCC with fewer BPP. In turn, HSC-1 and HSC-2 privilege efficiency over accuracy and result in lower PSNR compared to the baselines. Anyway, PSNR reduction is contained to a tolerable $-10 \%$ for the most aggressive quantization levels, in the face of an impressive improvement in the file size.

From Fig. 7 we also notice that both HSC and G-PCC methods can perform well in terms of symmetric Chamfer Distance when limiting the quantization level and the voxelization scaling factor, respectively.

Based on the previous results, we can conclude that the optimal trade-off between compression efficiency and accuracy in the reconstructed point cloud can be obtained with a quantization level of 9 or 10: in this range, the adoption of semantic segmentation in HSC- 2 can achieve up to $8 \times$ better compression than using G-PCC. In practice, inferring data labels and removing the least critical objects from the LiDAR point cloud before transmission guarantee quasi real-time operations as encoding and decoding times never exceed the LiDAR inter-frame time. Finally, even in the most aggressive, space-saving configuration (i.e., HSC-2), the PSNR is still guaranteed to be above $50 \mathrm{~dB}$ (a typical acceptable value for wireless transmission quality loss at which distortions in 


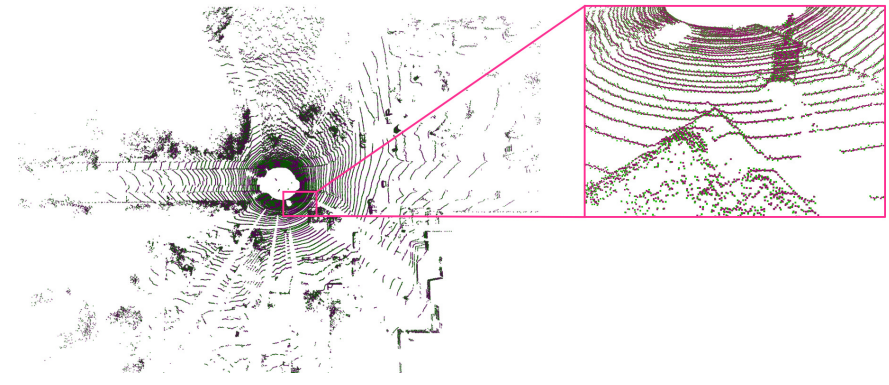

Fig. 8: Visual reconstruction of the point cloud obtained with HSC (green) vs. the raw LiDAR acquisition (violet) with quantization level equal to 10 .

compressed frames can be hardly noticed [26]) when BPP $>5$. This is also validated in Fig. 8, which shows how the reconstructed point cloud with quantization level equal to 10 is a good approximation of the original LiDAR acquisition.

\section{CONCLuding Remarks}

In this work we presented an innovative semantic compression algorithm for LiDAR point clouds, designed to achieve quasi real-time transmissions in autonomous driving scenarios. Our first contribution has been the interesting comparison between G-PCC and Draco for compression, which revealed how the former is not suitable for low-latency and real-time applications. We then proposed to enhance the compression performance by assisting Draco with RangeNet++ semantic segmentation as an approach to preemptively identify the least critical points and defer/cancel their transmissions in favor of more valuable data. We validated our framework on the Semantic KITTI dataset, and we showed that, even though there exists a trade-off between compression efficiency and accuracy, it is possible to compress the raw point cloud by up to 700 times to achieve real-time transmissions with tolerable accuracy degradation.

Future works will consider end-to-end system-level simulations to assess the benefits of the proposed framework in terms of network-related metrics such as the channel occupancy and the overall transmission latency.

\section{REFERENCES}

[1] N. Lu, N. Cheng, N. Zhang, X. Shen, and J. W. Mark, "Connected Vehicles: Solutions and Challenges," IEEE Internet of Things Journal, vol. 1, no. 4, pp. 289-299, Aug 2014.

[2] X. Chen, H. Ma, J. Wan, B. Li, and T. Xia, "Multi-view 3D object detection network for autonomous driving," in Proceedings of the IEEE Conference on Computer Vision and Pattern Recognition, 2017.

[3] C. Cao, M. Preda, and T. Zaharia, "3D point cloud compression: A survey," in 24th Conference on 3D Web Technology, 2019.

[4] J. Choi, V. Va, N. Gonzalez-Prelcic, R. Daniels, C. R. Bhat, and R. W. Heath, "Millimeter-Wave Vehicular Communication to Support Massive Automotive Sensing," IEEE Communications Magazine, vol. 54, no. 12, pp. 160-167, Dec. 2016.

[5] M. Giordani, A. Zanella, T. Higuchi, O. Altintas, and M. Zorzi, "On the Feasibility of Integrating mmWave and IEEE $802.11 \mathrm{p}$ for V2V Communications," IEEE Connected and Automated Vehicles Symposium (CAVS), 2018.

[6] T. Zugno, M. Drago, M. Giordani, M. Polese, and M. Zorzi, "Toward Standardization of Millimeter-Wave Vehicle-to-Vehicle Networks: Open Challenges and Performance Evaluation," IEEE Communications Magazine, vol. 58, no. 9, pp. 79-85, Sep. 2020.
[7] M. Giordani, A. Zanella, and M. Zorzi, "Millimeter wave communication in vehicular networks: Challenges and opportunities," in 6th International Conference on Modern Circuits and Systems Technologies (MOCAST), May 2017.

[8] R. Schnabel and R. Klein, "Octree-based point-cloud compression," in Proceedings of the 3rd Eurographics/IEEE VGTC conference on PointBased Graphics, 2006, pp. 111-121.

[9] L. Huang, S. Wang, K. Wong, J. Liu, and R. Urtasun, "OctSqueeze: Octree-Structured Entropy Model for LiDAR Compression," in Proceedings of the IEEE/CVF Conference on Computer Vision and Pattern Recognition, 2020, pp. 1313-1323.

[10] C. R. Qi, L. Yi, H. Su, and L. J. Guibas, "Pointnet++: Deep hierarchical feature learning on point sets in a metric space," in Advances in neural information processing systems, 2017, pp. 5099-5108.

[11] Google, "Draco 3D Data Compression," 2017, [Online]. Available: https://github.com/google/draco.

[12] A. Milioto, I. Vizzo, J. Behley, and C. Stachniss, "RangeNet++: Fast and accurate LiDAR semantic segmentation," in IEEE/RSJ International Conference on Intelligent Robots and Systems (IROS), 2019.

[13] M. Giordani, A. Zanella, T. Higuchi, O. Altintas, and M. Zorzi, "Investigating Value of Information in Future Vehicular Communications," IEEE Connected and Automated Vehicles Symposium (CAVS), 2019.

[14] T. Higuchi, M. Giordani, A. Zanella, M. Zorzi, and O. Altintas, "Valueanticipating V2V communications for cooperative perception," in IEEE Intelligent Vehicles Symposium (IV), Jun. 2019, pp. 1947-1952.

[15] D. Graziosi, O. Nakagami, S. Kuma, A. Zaghetto, T. Suzuki, and A. Tabatabai, "An overview of ongoing point cloud compression standardization activities: video-based (V-PCC) and geometry-based (GPCC)," APSIPA Transactions on Signal and Information Processing, vol. 9, Apr. 2020

[16] J. Behley, M. Garbade, A. Milioto, J. Quenzel, S. Behnke, C. Stachniss, and J. Gall, "SemanticKITTI: A dataset for semantic scene understanding of LiDAR sequences," in Proceedings of the IEEE International Conference on Computer Vision, 2019, pp. 9297-9307.

[17] B. Wu, A. Wan, X. Yue, and K. Keutzer, "Squeezeseg: Convolutional neural nets with recurrent CRF for real-time road-object segmentation from 3D LiDAR point cloud," in 2018 IEEE International Conference on Robotics and Automation (ICRA). IEEE, 2018, pp. 1887-1893.

[18] C. Xu, B. Wu, Z. Wang, W. Zhan, P. Vajda, K. Keutzer, and M. Tomizuka, "Squeezesegv3: Spatially-adaptive convolution for efficient point-cloud segmentation," arXiv preprint arXiv:2004.01803, 2020.

[19] Y. Zhou and O. Tuzel, "Voxelnet: End-to-end learning for point cloud based 3D object detection," in Proceedings of the IEEE Conference on Computer Vision and Pattern Recognition, 2018, pp. 4490-4499.

[20] J. Ballé, D. Minnen, S. Singh, S. J. Hwang, and N. Johnston, "Variational image compression with a scale hyperprior," in International Conference on Learning Representations, 2018.

[21] Y. Zhang, Z. Zhou, P. David, X. Yue, Z. Xi, B. Gong, and H. Foroosh, "PolarNet: An Improved Grid Representation for Online LiDAR Point Clouds Semantic Segmentation," in Proceedings of the IEEE/CVF Conference on Computer Vision and Pattern Recognition, 2020, pp. 9601-9610.

[22] T. Cortinhal, G. Tzelepis, and E. E. Aksoy, "SalsaNext: Fast, Uncertainty-aware Semantic Segmentation of LiDAR Point Clouds for Autonomous Driving," arXiv preprint arXiv:2003.03653, 2020.

[23] O. Devillers and P. . Gandoin, "Geometric compression for interactive transmission," in Proceedings Visualization, 2000, pp. 319-326.

[24] M. Giordani, A. Zanella, T. Higuchi, O. Altintas, and M. Zorzi, "A framework to assess Value of Information in future vehicular networks," 1st MobiHoc Workshop on Technologies, mOdels, and Protocols for Cooperative Connected Cars (TOP-Cars), Jul. 2019.

[25] D. Tian, H. Ochimizu, C. Feng, R. Cohen, and A. Vetro, "Geometric distortion metrics for point cloud compression," in IEEE International Conference on Image Processing (ICIP), 2017.

[26] V. V. Abramova, S. K. Abramov, V. V. Lukin, K. O. Egiazarian, and J. T. Astola, "On required accuracy of mixed noise parameter estimation for image enhancement via denoising," EURASIP Journal on Image and Video Processing, vol. 2014, no. 1, p. 3, Jan. 2014. 